\title{
BMI open Use of cardiocerebral resuscitation or AHA/ERC 2005 Guidelines is associated with improved survival from out-of-hospital cardiac arrest: a systematic review and meta-analysis
}

\author{
Marcus Salmen, ${ }^{1}$ Gordon A Ewy, ${ }^{2}$ Comilla Sasson ${ }^{3}$
}

To cite: Salmen M, Ewy GA, Sasson C. Use of cardiocerebral resuscitation or AHA/ERC 2005 Guidelines is associated with improved survival from out-of-hospital cardiac arrest: a systematic review and meta-analysis. BMJ Open 2012;2:e0001273. doi:10.1136/bmjopen-2012001273

- Prepublication history and additional material for this paper are available online. To view these files please visit the journal online (http://dx. doi.org/10.1136/bmjopen2012-001273)

Received 25 April 2012 Accepted 28 August 2012

This final article is available for use under the terms of the Creative Commons Attribution Non-Commercial 2.0 Licence; see http://bmjopen.bmj.com

For numbered affiliations see end of article

Correspondence to Dr Marcus Salmen; marcus.salmen@hcmed.org

\section{ABSTRACT}

Objective: To determine whether the use of cardiocerebral resuscitation (CCR) or AHA/ERC 2005

Resuscitation Guidelines improved patient outcomes from out-of-hospital cardiac arrest (OHCA) compared to older guidelines.

Design: Systematic review and meta-analysis.

Data sources: MEDLINE, EMBASE, Web of Science and the Cochrane Library databases. We also hand-searched study references and consulted experts.

Study selection: Design: randomised controlled trials and observational studies.

Population: OHCA patients, age $>17$ years.

Comparators: 'Control' protocol versus 'Study' protocol. 'Control' protocol defined as AHAJERC 2000 Guidelines for cardiopulmonary resuscitation (CPR). 'Study' protocol defined as AHA/ERC 2005 Guidelines for CPR, or a CCR protocol.

Outcome: Survival to hospital discharge.

Quality: High-quality or medium-quality studies, as measured by the Newcastle Ottawa Scale using predefined categories.

Results: Twelve observational studies met inclusion criteria. All the three studies using CCR demonstrated significantly improved survival compared to use of AHA 2000 Guidelines, as did five of the nine studies using AHA/ERC 2005 Guidelines. Pooled data demonstrate that use of a CCR protocol has an unadjusted OR of 2.26 $(95 \% \mathrm{Cl} 1.64$ to 3.12$)$ for survival to hospital discharge among all cardiac arrest patients. Among witnessed ventricular fibrillation/ventricular tachycardia (VFNT) patients, CCR increased survival by an OR of 2.98 (95\% $\mathrm{Cl} 1.92$ to 4.62). Studies using AHA/ERC 2005 Guidelines showed an overall trend towards increased survival, but significant heterogeneity existed among these studies.

Conclusions: We demonstrate an association with improved survival from OHCA when CCR protocols or AHA/ERC 2005 Guidelines are compared to use of older guidelines. In the subgroup of patients with witnessed VF/ $\mathrm{VT}$, there was a threefold increase in $\mathrm{OHCA}$ survival when CCR was used. CCR appears to be a promising resuscitation protocol for Emergency Medical Services providers in increasing survival from OHCA. Future research will need to be conducted to directly compare AHA/ERC 2010 Guidelines with the CCR approach.

\section{ARTICLE SUMMARY}

Article focus

- This review investigates whether use of newer resuscitation protocols-AHA/ERC 2005 Guidelines and cardiocerebral resuscitation (CCR)-has improved survival from out-of-hospital cardiac arrest.

Key messages

- Twelve observational studies on the topic were found, including three studies in which CCR was used.

- When AHA/ERC 2005 Guidelines were compared with AHA/ERC 2000 Guidelines, use of the newer guidelines showed an overall trend towards improved survival.

- CCR was associated with a significant survival benefit $(\mathrm{OR}=2.26)$ compared to older guidelines, including a threefold increase in survival for patients with a witnessed ventricular fibrillation/ ventricular tachycardia arrest.

Strengths and limitations of this study

- This is the first systematic review and meta-analysis of the current evidence on the use of CCR and updated AHA/ERC Guidelines.

- Only observational studies could be found and reported in this review.

- Significant heterogeneity existed among the included studies in exact resuscitation and treatment protocols, limiting the pooling of data and comparisons between studies.

\section{INTRODUCTION}

Out-of-hospital cardiac arrest (OHCA) is a major cause of global mortality and a significant public health concern. In the USA alone, an estimated 300000 people suffer an OHCA each year, and only $7 \%$ will survive to hospital discharge. ${ }^{1}$ The survival rate had previously remained unchanged for over three decades despite a dramatic increase in our understanding of OHCA and its predictors of survival. ${ }^{2}$ 
International standards for the emergency treatment of OHCA have existed since 1966, and the American Heart Association (AHA) published its first set of formal guidelines for cardiopulmonary resuscitation (CPR) in 1992. ${ }^{34}$ Updates to the AHA and European Resuscitation Council (ERC) Guidelines in 2000 and 2005 reflected, in large part, a growing understanding of the electrophysiological phases of cardiac arrest and the need to minimise interruptions in chest compressions. ${ }^{5}{ }^{6}$ Concurrently, animal and human studies have indicated that active ventilation during resuscitation may be unnecessary, and even detrimental. ${ }^{78}$ This has lead to the development of a new body of Emergency Medical Services (EMS) resuscitation protocols that minimise ventilation and maximise the amount of time spent by EMS doing chest compressions. One such protocol is cardiocerebral resuscitation (CCR), which emphasises passive ventilation, continuous chest compressions and delayed intubation. CCR was first instituted in 2003 as an alternative resuscitation protocol due to poor survival rates observed using the standard guidelines. ${ }^{9} 10$ At the time, CCR represented a significant departure from the AHA 2000 Guidelines because of its use of a single defibrillator shock instead of triple-stacked shocks. This key resuscitation element was later incorporated into AHA and ERC 2005 Guidelines, and CCR has since shown promise in improving survival from OHCA. ${ }^{11} 12$

Although a majority of EMS systems continue to use AHA-based or ERC-based guidelines for CPR, a growing number have adopted the CCR protocol. To date, no systematic review has been conducted to compare standard AHA/ERC Guidelines to CCR protocols. Although the AHA Guidelines have several minor differences from the ERC Guidelines, we considered them similar enough in their strategies for ventilations, compressions and defibrillations to be grouped together for the purposes of this review. This is the first systematic review and meta-analysis of the literature to examine the OHCA survival impact of EMS transitions from use of AHA/ERC 2000 Guidelines to use of a CCR protocol or AHA/ERC 2005 Guidelines.

\section{METHODS}

This systematic review and meta-analysis were performed according to guidelines set forth by the MOOSE (Meta-Analysis Of Observational Studies in Epidemiology) checklist. ${ }^{13}$ Planning and study design were done by two authors (MS and CS), and included creation of an electronic database (Microsoft Excel) with defined primary endpoints and variables of interest that can be obtained from study authors on request.

\section{Search strategy}

A search was conducted of the following databases: MEDLINE (Ovid) (via PubMed), 1950 to December 2011; EMBASE (Ovid), 1966-2011; Web of Science, 1970-2011; the Cochrane Central Register of Controlled Trials; the Cochrane Database of Systematic Reviews; the Database of Abstracts of Reviews of Effects; the NHS Economic
Evaluation Database and the Health Technology Assessment Database. Our search strategy included searching for keywords such as 'cardiocerebral', 'minimally interrupted' and 'continuous compressions' (using truncation and adjacency techniques) from within the subject headings of 'Heart Arrest', 'Resuscitation' and 'Emergency Medical Services'. Results were limited to human studies with English abstracts published since 2000. The full details of all the search strategies can be obtained from study authors on request. Additionally, we reviewed the bibliographies of included studies and published review articles, as well as sought expert opinion (GE) to obtain additional studies. The search included all through 1 December 2011.

\section{Inclusion criteria}

Studies were considered eligible for inclusion if they met criteria for study design, control protocol, study protocol, outcome and methodological quality (box 1). Similar to other recent studies, ${ }^{14}$ methodological quality was assessed using the Newcastle Ottawa Scale (NOS) for cohort studies, wherein studies are given up to nine total points for fulfilling criteria in selection, comparability and exposure. ${ }^{15}$ We categorised studies as high quality if they received eight or nine points, medium quality if they received six or seven points, and low quality if they received five points or less. ${ }^{14}$ Only high-quality and medium-quality studies were included in this review.

We excluded reviews, editorials, opinions, studies on bystander resuscitation, studies comparing the absence/ presence of advanced life support, studies focusing on mechanical devices and studies available only as meeting abstracts. In addition, we excluded studies with non-English language full-text, studies meeting incomplete criteria for the resuscitation protocols and studies containing duplicative data of the included studies.

To be considered for inclusion, studies must have explicitly stated use of AHA 2000 Guidelines or ERC 2000 Guidelines as their EMS 'control' protocol. These studies had to directly compare EMS use of AHA 2000/ERC 2000 Guidelines to either AHA 2005, ERC 2005 or CCR

\section{Box 1 Inclusion criteria for eligible studies}

Study design: randomised controlled trials and observational studies.

- Population: out-of-hospital cardiac arrest patients, >17 years of age.

- EMS Control Protocol: AHA 2000 guidelines or ERC 2000 Guidelines for cardiopulmonary resuscitation.

- EMS Study Protocol: AHA 2005 Guidelines or ERC 2005 Guidelines for cardiopulmonary resuscitation, or cardiocerebral resuscitation

- Outcomes: rate of bystander cardiopulmonary resuscitation, Emergency Medical Services response time, proportion of initial VF/VT and survival to hospital discharge.

- Quality: high or medium quality, as categorised using the Newcastle Ottawa Scale for cohort studies. 
Figure 1 Flow chart depicting the outline of study selection process.

\begin{tabular}{|c|c|c|c|}
\hline & Compressions & $\underline{\text { Ventilation }}$ & Defibrillation \\
\hline $\begin{array}{l}\text { AHA } 2000 \\
\text { CPR }\end{array}$ & $15: 2$ ratio & $\begin{array}{l}\text { Tracheal Intubation } \\
10-12 \text { breaths/min }\end{array}$ & $\begin{array}{l}\text { Pre: --- } \\
\quad \text { * Triple Shock * } \\
\quad \text { * Pulse Check* } \\
\text { Post: } 1 \text { min CPR }\end{array}$ \\
\hline $\begin{array}{l}\text { ERC } 2000 \\
\text { CPR }\end{array}$ & $15: 2$ ratio & $\begin{array}{l}\text { Tracheal Intubation } \\
\sim 12 \text { breaths/min }\end{array}$ & $\begin{array}{l}\text { Pre: -- } \\
\quad \text { * Triple Shock* } \\
\text { Post: } 1 \text { min CPR } \\
\quad \text { * Pulse Check* }\end{array}$ \\
\hline $\begin{array}{l}\text { AHA } 2005 \\
\quad \text { CPR }\end{array}$ & $30: 2$ ratio & $\begin{array}{l}\text { Tracheal Intubation } \\
8-10 \text { breaths/min }\end{array}$ & $\begin{array}{l}\text { Pre: Optional } 5 \text { cyc } \\
\quad \text { * Single Shock* } \\
\text { Post: } 5 \text { cycles } \\
\text { * Pulse Check* }\end{array}$ \\
\hline $\begin{array}{l}\text { ERC } 2005 \\
\quad \text { CPR }\end{array}$ & $30: 2$ ratio & $\begin{array}{l}\text { Tracheal Intubation } \\
8-10 \text { breaths/min }\end{array}$ & $\begin{array}{l}\text { Pre: } 2 \text { min CPR } \\
\text { * Single Shock* } \\
\text { Post: } 2 \text { min CPR } \\
\text { * Pulse Check* }\end{array}$ \\
\hline $\begin{array}{c}\text { Cardiocerebral } \\
\text { Resuscitation (2003) }\end{array}$ & $\begin{array}{l}\text { Continuous } \\
100 / \mathrm{min}\end{array}$ & $\begin{array}{c}\text { Passive Only; } \\
\text { Delayed Intubation }\end{array}$ & $\begin{array}{l}\text { Pre: } 200 \text { compress } \\
\text { * Single Shock* } \\
\text { Post: } 200 \text { compress } \\
\text { * Pulse Check * }\end{array}$ \\
\hline
\end{tabular}

protocols. CCR has several distinct and important differences from the AHA and ERC Guidelines for CPR, which are illustrated in figure 1. We defined an EMS study protocol to be a CCR protocol if it contained four of five critical elements 12: (1) 200 chest compressions upon initial EMS arrival; (2) if indicated, administration of a single, direct shock; (3) immediate postshock 200 chest compressions; (4) delayed intubation until after three full cycles and (5) administration of epinephrine as soon as possible.

\section{Study selection process}

We screened citations in three stages (figure 2). In Stage 1, one reviewer (MS) reviewed all citations by title or abstract to exclude clearly irrelevant and duplicate citations. In Stage 2, two reviewers (MS and CS) screened remaining abstracts for potential inclusion. In Stage 3, three reviewers (MS, CS and GE) reviewed the full text of all potentially eligible studies, and final inclusion was based on the agreement of all three investigators. Reviewers were not blinded to study authors or outcomes.

\section{Data extraction}

Relevant information from the studies, including characteristics of the study population and outcome measures, was extracted by two reviewers (MS and CS) using the prepared extraction database (Microsoft Excel). Data on study characteristics (year and location), variables of interest (age, gender, witnessed arrest, bystander CPR, initial shockable rhythm and EMS response time), total patient numbers per group and outcomes of relevant groups were extracted. When included, data were extracted directly from Utstein templates. Absolute numbers were recalculated when percentages were reported.

\section{Data analysis and synthesis}

Individual study data were used to calculate unadjusted OR for survival to hospital discharge for each EMS 'control' and EMS 'study' (defined as either AHA 2005 or
CCR) protocol. Outcome data of the included studies were combined within each group to estimate the pooled effect (OR) for the use of CCR compared to AHA 2000 and the use of AHA 2005 compared to AHA 2000. Because witnessed ventricular fibrillation is a commonly used measure of OHCA survival, we conducted a subgroup analysis of these patients to compare the control versus study protocols for AHA 2005 and CCR guidelines. Calculations were based on a DerSimonian and Laird random effects model. ${ }^{16}$ Heterogeneity among trials was quantified with Higgins's and Thompson's ${ }^{2}$, which can be interpreted as the percentage of variability due to heterogeneity between studies rather than sampling error. Publication bias was assessed by generating separate funnel plots for each of the two groups of studies. Findings are presented as point estimates and 95\% CIs, and when appropriate, weight of individual studies. All analyses were performed within RevMan V.5.1. ${ }^{17}$

\section{RESULTS}

\section{Identification and selection of studies}

Our initial electronic search yielded 654 citations, and an additional 27 were identified through hand-search of bibliographies and other sources. After an initial screen of titles, 100 abstracts were reviewed, and 22 of those were subsequently selected for full-text review. Six studies were excluded for lacking sufficient number of CCR elements, three studies excluded for sharing duplicative data with included studies and one study for non-English language text. Finally, 12 total studies satisfied the predetermined inclusion criteria: three using CCR and nine using AHA 2005 or ERC 2005 Guidelines, as shown in figure 2 .

\section{Characteristics of included studies}

The final sample included 12 studies, with approximately 19634 subjects. Study characteristics and 
Figure 2 Forest plot of pooled ORs: cardiocerebral resuscitation versus AHA 2000 studies.

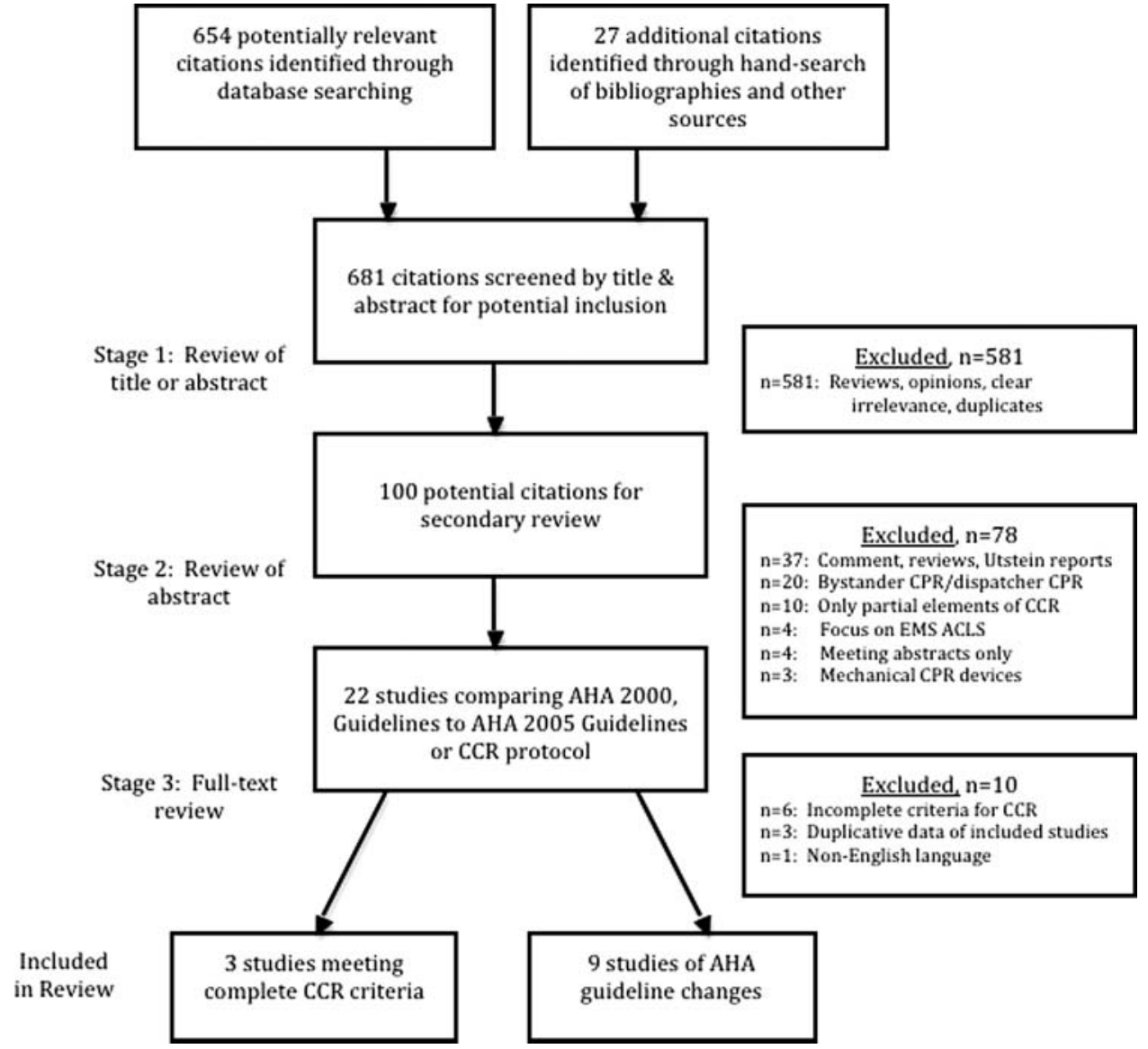

654 potentially relevant ations identified through entified through hand-search of bibliographies and other sources 37: Comment reviews, Utstein report $n=20$ : Bystander CPR/dispatcher CPR artial elements of CC.

: Focus on EMS ACL.S

=4: Meeting abstracts only $n=3$ : Mechanical CPR devices $n=3$ : Duplicative data of included studies $n=1$ : Non-English language pertinent outcome data are shown in table 1 . All 12 articles that were included were prospective observational studies with data on survival preimplementation and postimplementation of guidelines. Eight of the 12 studies were rated as high quality, and four were rated as medium quality. No randomised controlled trials were identified that met inclusion criteria.

Three studies, which included a total of 2820 subjects, met the predetermined criteria for use of AHA 2000 (control) versus CCR (study) protocol. ${ }^{18-20}$ Nine studies, which included a total of 16814 subjects, compared the use of AHA 2000/ERC 2000 (control) versus AHA 2005/ERC 2005 (study) protocols. ${ }^{21-29}$

Eight of the studies were conducted in North America, and one each in Taiwan, Norway, New Zealand and Denmark. Data on EMS control protocols (2000 Guidelines) were collected in the range of 2003-2006, except for Kellum (2008) which began collecting data in 2001. Data on EMS study protocols (CCR or AHA/ ERC 2005 Guidelines) were collected in the range of 2004-2008. Of note, none of the studies using CCR, but five of studies using AHA 2005 Guidelines explicitly reported consistent use of therapeutic hypothermia during the study period. ${ }^{21} 23252628$

The mean subject age across all studies was 64 years, and the mean gender distribution was $66.4 \%$ male. Two studies had significant differences in age between their control and study populations. ${ }^{22} 25$
There was a wide range across studies in the proportion of OHCA receiving bystander CPR (8-58\%) with a mean of $34.6 \%$. The mean EMS response time was $6.2 \mathrm{~min}$, with a range of 4.7-9.3 min. The mean proportion of OHCA patients with a presenting rhythm of ventricular fibrillation or ventricular tachycardia (VF/VT) was $29.9 \%$, with a range of $5.6-50 \%$. Three studies reported statistically significant differences in the proportion of witnessed arrest, proportion of bystander-CPR, or response time between control and study populations. ${ }^{20} 2223$

\section{Overall outcomes}

Survival to hospital discharge was identified as the primary or secondary outcome in all studies. Mean survival when using AHA/ERC 2000 Guidelines was 6.9\% (range of $1.8-11.1 \%$ ). Mean survival to discharge increased to $10.1 \%$ (6.5-19.4\%) when using AHA/ERC 2005 Guidelines, and to 9.3\% (5.4-18.3\%) when CCR was used. Overall mean survival across all studies and protocols was $9.7 \%$.

\section{AHA 2000 Guidelines as compared to CCR}

All three of the studies using a CCR protocol showed significantly improved survival for CCR compared to use of AHA 2000 Guidelines. Pooled analysis of data from these studies (figure 3) demonstrated a statistically significant survival benefit when using a CCR protocol, with a pooled OR of 2.26 (95\% CI 1.64 to 3.12). 
Table 1 Characteristics and outcomes of included studies

\begin{tabular}{|c|c|c|c|c|c|c|c|c|}
\hline Study & Location & Quality* $^{*}$ & $\begin{array}{l}\text { Control } \\
\text { versus } \\
\text { study } \\
\text { protocol }\end{array}$ & $\begin{array}{l}\text { Bystander } \\
\text { CPR (\%) }\end{array}$ & $\begin{array}{l}\text { Response } \\
\text { time (min) }\end{array}$ & $\begin{array}{l}\text { Initial } \\
\text { VF/VT } \\
(\%)\end{array}$ & $\begin{array}{l}\text { Total } \\
\text { arrests } \\
\text { (n) }\end{array}$ & $\begin{array}{l}\text { Survival to } \\
\text { discharge } \\
(n, \%)\end{array}$ \\
\hline \multicolumn{9}{|c|}{ Cardiocerebral resuscitation } \\
\hline \multirow[t]{2}{*}{ Bobrow et $a l^{18}$} & Arizona & High & AHA 2000 & 34 & 5.6 & 31.7 & 218 & $4(1.8 \%)$ \\
\hline & & & MICR† & 39 & 5.2 & 29.9 & 668 & $36(5.4 \%)$ \\
\hline \multirow[t]{2}{*}{ Garza et al ${ }^{19}$} & Missouri & High & AHA 2000 & 38 & 5.4 & 31.4 & 1097 & $64(5.8 \%)$ \\
\hline & & & $\begin{array}{l}\mathrm{KCMO} \\
\text { Protocol‡ }\end{array}$ & 47 & 5.3 & 33.3 & 339 & $37(10.9 \%)$ \\
\hline \multirow[t]{2}{*}{ Kellum et $a p^{0}$} & Wisconsin & High & AHA 2000 & $45 \S$ & $7.4 \S$ & 42.5 & 268 & $21(7.8 \%)$ \\
\hline & & & CCR & $45 \S$ & $8.6 \S$ & 47.4 & 230 & $42(18.3 \%)$ \\
\hline \multicolumn{9}{|c|}{ AHA/ERC 2005 guidelines } \\
\hline \multirow[t]{2}{*}{ Aufderheide et $a f^{1}$} & MN, TX, & High & AHA 2000 & 38 & 5.6 & 25.3 & 1641 & $166(10.1 \%)$ \\
\hline & $\mathrm{NE}, \mathrm{FL}, \mathrm{NC}$ & & AHA 2005 & 40 & 5.6 & 23.9 & 1605 & $211(13.1 \%)$ \\
\hline \multirow{2}{*}{ Bigham et $a^{22}$} & Canada & Medium & AHA 2000 & 29 & 5.8 & 23.1 & 5054 & 294 (5.8\%) \\
\hline & and USA & & AHA 2005 & 35 & 5.7 & 24.2 & 2725 & 177 (6.5\%) \\
\hline \multirow[t]{2}{*}{ Hinchey et $a^{23}$} & North & High & AHA 2000 & 39 & 6.1 & 29.3 & 425 & $18(4.2 \%)$ \\
\hline & Carolina & & AHA 2005ף & 35 & 5.4 & 24.1 & 410 & 47 (11.5\%) \\
\hline \multirow[t]{2}{*}{ Hung et $a$ f4 $^{4}$} & Taiwan & High & AHA 2000 & 8 & 4.7 & 5.6 & 463 & 47 (10.2\%) \\
\hline & & & AHA 2005 & 10 & 4.7 & 7.2 & 430 & $30(7.0 \%)$ \\
\hline \multirow[t]{2}{*}{ Lick et $a^{25}$} & Minnesota & High & $\mathrm{AHA} 2000^{\star *}$ & 20 & 7.5 & 27.3 & 106 & $9(8.5 \%)$ \\
\hline & & & $\mathrm{AHA} 2005^{\star \star}$ & 29 & 7.2 & 36.4 & 247 & 48 (19.4\%) \\
\hline \multirow[t]{2}{*}{ Olasveengen et $a^{26}$} & Norway & Medium & ERC 2000 & 52 & 8 & 35 & 435 & 46 (10.6\%) \\
\hline & & & ERC 2005 & 58 & 9 & 34 & 482 & $63(13.1 \%)$ \\
\hline \multirow[t]{2}{*}{ Robinson et $a^{{ }^{7}}$} & New & Medium & AHA 2000 & 50 & 9.1 & 45 & 162 & 18 (11.1\%) \\
\hline & Zealand & & AHA 2005 & 51 & 9.3 & 50 & 170 & 20 (11.8\%) \\
\hline \multirow[t]{2}{*}{ Sayre et $a^{28}$} & Ohio & High & AHA $2000+\dagger$ & 28 & 5.1 & 21.7 & 660 & $40(6.1 \%)$ \\
\hline & & & AHA 2005†† & 28 & 5.1 & 22.3 & 1021 & $96(9.4 \%)$ \\
\hline \multirow[t]{2}{*}{ Steinmetz et $a^{29}$} & Denmark & Medium & ERC 2000 & 24 & 5 & 34.1 & 193 & $21(10.9 \%)$ \\
\hline & & & ERC 2005ł‡ & 28 & 5 & 43.3 & 226 & $41(18.1 \%)$ \\
\hline \multicolumn{9}{|c|}{ 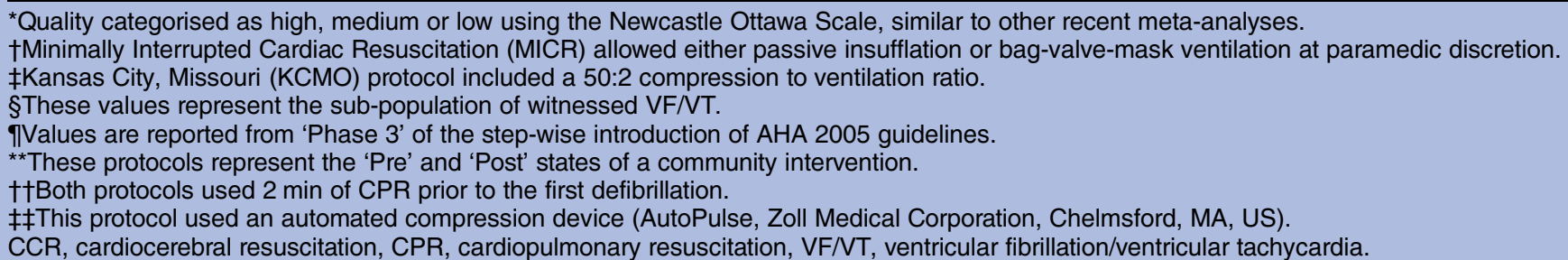 } \\
\hline
\end{tabular}

Heterogeneity among the studies was not significant $\left(\mathrm{I}^{2}=0.0 \%, \mathrm{p}=0.61\right)$.

Subgroup analysis of the patients with a presenting rhythm of witnessed VF/VT showed a significant association with survival for the CCR protocol as compared to the AHA 2000 Guidelines. Pooled OR of survival to hospital discharge for this subgroup was 2.98 (95\% CI 1.92 to 4.62) (figure 3). The funnel plot of these three studies was not suggestive of publication bias (see online supplementary figure $\mathrm{S} 1$ ).

\section{AHA/ERC 2000 Guidelines as compared to AHA/ERC 2005 Guidelines}

Eight of the nine studies using AHA/ERC 2005 Guidelines demonstrated improved survival to hospital discharge for use of AHA/ERG 2005 compared to AHA/ERC 2000 Guidelines, a result that was statistically significant in five out of nine studies (figure 4).
Although the overall trend was towards a survival benefit for AHA/ERC 2005 Guidelines, heterogeneity tests revealed significant heterogeneity among these nine studies $\left(\mathrm{I}^{2}=72 \%, \mathrm{p}=0.0004\right)$, so a pooled OR for survival was not calculated (figure 5). ${ }^{30}$

Subgroup analysis of the patients with a presenting rhythm of witnessed VF/VT showed a trend towards improved survival for the AHA/ERC 2005 Guidelines as compared to the AHA/ERC 2000 Guidelines. However, there was significant heterogeneity between studies so a pooled OR was not calculated. ${ }^{30}$ The funnel plot of these nine studies was not suggestive of publication bias (see online supplementary figure S2).

\section{DISCUSSION}

Main findings

In our systematic review of 12 studies and 19634 subjects, we found a consistent and significant survival benefit 
Figure 3 Forest plot of ORs for witnessed ventricular fibrillation/ ventricular tachycardia survival: cardiocerebral resuscitation versus AHA/ERC 2000 studies.

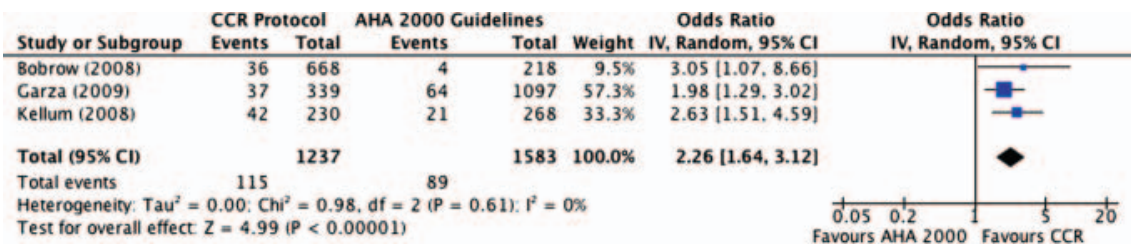

However, the most significant difference between AHA/ERC 2005 Guidelines and CCR protocols is the decision to delay intubation in favour of passive ventilation. An existing body of studies has shown that intubations can (1) cause significant delays and/or interruptions in chest compressions, ${ }^{38}$ and (2) lead to excessive positive pressure ventilation which can reduce venous return, reduce cerebral and coronary perfusion pressures and consequently reduce likelihood of survival. $^{78}$

The benefit of passive insufflation was recently shown in a subanalysis of the 'minimally interrupted cardiac resuscitation' protocol used in Arizona and included in this meta-analysis through Bobrow et al. ${ }^{39}$ Owing to the reluctance of EMS teams to forgo active ventilation, this protocol allowed airway management through either passive insufflation or bag-valve-mask ventilation, at the discretion of the paramedics. For the subset of patients most likely to survive (witnessed VF/VT), neurologically intact survival was significantly higher among patients who received passive insufflation only as compared to bag-valve mask (38.2\% compared to $25.8 \%$ ). ${ }^{39}$

Another study, published in 2011, showed an association with increased survival when a non-standard resuscitation protocol was implemented in Sussex County, UK. ${ }^{40}$ Delayed ventilation was a key feature in this protocol; patients with witnessed OHCA and initial VF/VT were not given any active ventilations until after three cycles of 100 compressions plus shock. Using this strategy, survival in these patients increased from a historical baseline of $13 \%$ to approximately $30 \%$. $^{40}$

The newest ERC and AHA Guidelines were recently released in late-2010. ${ }^{41}{ }^{42}$ The ERC Guidelines have removed the recommendation for a specified period of CPR before first defibrillation, while the AHA Guidelines have a strong emphasis on $\mathrm{C}-\mathrm{A}-\mathrm{B}$ : compressions, airway and then breathing. The shift to focusing on providing quality chest compression at a rate of at least $100 / \mathrm{min}$ is a change from the AHA 2000 and 2005 Guidelines in which the airway and breathing were first addressed.
Figure 4 Forest plot of ORs: AHA/ERC 2005 versus AHA/ERC 2000 studies.

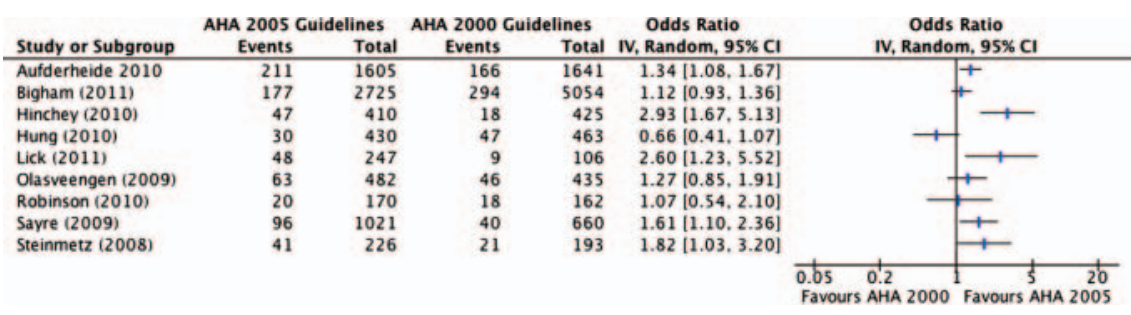


Figure 5 Forest plot of ORs: AHA/ERC 2005 versus AHA/ERC 2000 studies.

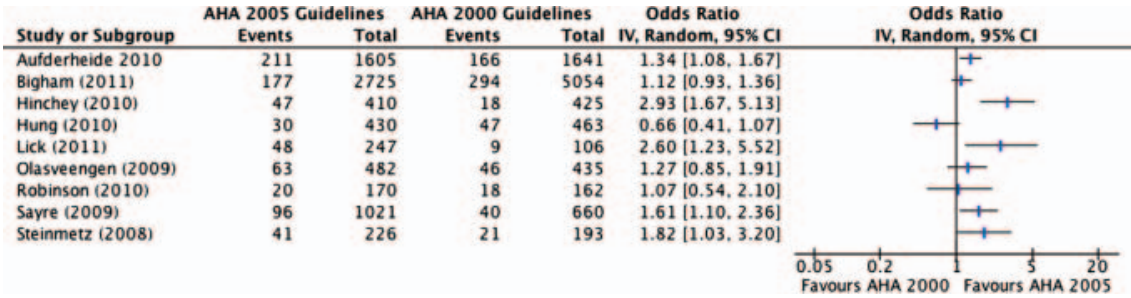

Although this is similar to the approach to CCR, there are still significant differences, the most significant being that CCR encourages compressions without ventilations. Future research will need to be conducted to directly compare survival between use of the updated AHA and ERC 2010 Guidelines and use of CCR.

\section{Limitations}

This systematic review yielded a total of 12 studies reporting results on CCR and AHA/ERC 2005 Guidelines as compared to AHA/ERC 2000 Guidelines. This relatively small sample limits the generalisability of our results in several ways. Only three studies reported results from the use of CCR protocols, which limited the meta-analysis to include only 2820 total subjects. Additionally, some of the included studies reported few total patients surviving to hospital discharge, and thus were not as heavily weighted in the meta-analysis. However, even with a small number of studies and total subjects, this meta-analysis was sufficiently powered to demonstrate a significant survival benefit. Finally, although our funnel plots did not suggest a significant publication bias, it is possible that unpublished, negative studies were underrepresented in our review. Future research from multiple research centres across the USA and internationally could potentially strengthen the results demonstrated here.

All of the included studies report data from observational studies; no randomised-controlled trials were found that met inclusion criteria, and thus this review was unable to locate studies of the highest scientific quality. Indeed, randomisation of OHCA patients to different EMS protocols is impractical and ethically problematic. All of the observational studies included in this review were found to be of high or medium methodological quality based on the NOS, but owing to inherent weaknesses in observational studies, there exists the potential for confounding and bias within these studies. The combination of multiple observational studies into a meta-analysis has the potential limitation of masking confounders that exist within the individual studies. These confounders may include a number of unrecognised variables of cardiac resuscitation, as well as possible changes made to the emergency healthcare systems between the 'control' and 'study' protocols, such as community education or improved quality of chest compressions by EMS. Additionally, it is possible that the survival improvement demonstrated here with newer protocols is confounded by temporal changes in the communities studied. Changes in community demographics or disease burden that occurred between and within the periods of data collection could have contributed to the observed changes in survival. The overall similarity in demographics and characteristics of the 'control' and 'study' subjects (as reported in the individual studies) makes it unlikely that this potential confounder has had a significant influence on our overall results. However, the existence of other, unrecognised confounders of the individual studies remains an important limitation to our meta-analysis.

The introduction of therapeutic hypothermia represents another important possible limitation to the interpretation of these results. The use of postarrest hypothermia was not consistent between 'control' and 'study' protocols of individual studies. Four of the nine studies using AHA 2005 Guidelines reported significantly increased use of therapeutic hypothermia during the 'study' protocol period (two other studies did not mention change in hypothermia use). Given the strong evidence for the benefit of therapeutic hypothermia, ${ }^{43} 44$ it is thus likely that the use of hypothermia contributed to the overall trend towards improved survival; our analysis is limited in its ability to calculate the magnitude of that contribution. However, therapeutic hypothermia was not used on any of the patients included in the three CCR studies. Thus, it is extremely unlikely that hypothermia alone could be an alternative explanation for the increase in survival demonstrated by use of the CCR protocols.

The heterogeneity of the nine studies using AHA 2005 or ERC 2005 guidelines precluded pooling of their data and a summary estimate of effect. These studies were also heterogeneous in the specifics of the resuscitation protocols used, many employing minor deviations from the AHA or ERC guidelines. For example, both the 'control' and 'study' protocols of Sayre et al. (2009) utilised $2 \mathrm{~min}$ of CPR before first defibrillation attempt, and Steinmetz et al. (2009) utilised a mechanical compression device. Additionally, there exist minor but important differences between the AHA and ERC Resuscitation Guidelines. While these variations might have influenced the results of the individual studies, the random-effects model used for our pooled data analysis accounted for some of this variability. Still these variations serve to highlight the difficulty of standardising resuscitation protocols and comparing EMS systems.

Although delayed intubation is one of the pillars of CCR, the three studies included in the "AHA 2000 as 
compared to CCR' group all utilised different protocols for airway management during the 'study' protocol time period. The Kansas City Missouri (KCMO) protocol used in Garza et al. (2009) used a 50:2 compression to the ventilation ratio. Kellum et al. (2010) delayed intubation for patients with shockable rhythms only. Most significantly, Bobrow et al. (2008) allowed bag-valve-mask ventilation at the paramedics' discretion. The differences in the airway management strategies of these three studies make it more difficult to assess which aspects of the CCR protocol contributed most to increased survival, and limits the generalisability of our results.

\section{CONCLUSIONS}

The results of this systematic review and meta-analysis demonstrate an association with survival from OHCA when CCR protocols or AHA/ERC 2005 Guidelines are compared to AHA/ERC 2000 Guidelines. Although no randomised trials have yet been reported, multiple high-quality observational studies have demonstrated an association with increased survival post-AHA/ERC 2005 guideline or CCR implementation. Additionally, in the pooled subgroup of witnessed VF/ VT patients, there was a threefold increase in OHCA survival after a CCR protocol was implemented. The focus of the updated AHA 2010 and ERC 2010 Guidelines shifts emphasis towards providing uninterrupted, quality chest compressions. However, these changes still do not incorporate key elements of the CCR protocol that this study indicates are associated with improved OHCA survival, such as passive insufflation and minimal interruptions of chest compressions. Our study suggests that CCR appears to be a promising resuscitation protocol for EMS providers in increasing survival from OHCA. Future research will need to be conducted to directly compare AHA/ERC 2010 Guidelines with the CCR approach.

\section{Author affiliations}

${ }^{1}$ Department of Emergency Medicine \& Internal Medicine, Hennepin County Medical Center, Minneapolis, Minnesota, USA

${ }^{2}$ Department of Medicine, University of Arizona Sarver Heart Center, University of Arizona College of Medicine, Tucson, Arizona, USA

${ }^{3}$ Department of Emergency Medicine, University of Colorado, Aurora, Colorado, USA

Contributors MS and CS conceptualised and designed this systematic review and meta-analysis. MS and CS were substantially involved in data acquisition (literature search, study selection and data abstraction). MS and CS performed the analyses and were substantially involved in data interpretation. MS, GE and CS provided data used for the analysis and relevantly contributed to the interpretation and intellectual content of the manuscript. MS and CS drafted the manuscript. All authors revised the manuscript critically for important intellectual content. All authors approved the final version.

Competing interests None.

Provenance and peer review Not commissioned; externally peer reviewed.

Data sharing statement Available from authors upon request.

\section{REFERENCES}

1. Roger VL, Go AS, Lloyd-Jones DM, et al. Heart disease and stroke statistics-2011 update: a report from the American Heart Association. Circulation 2011;123:e18-209.
2. Sasson C, Rogers MA, Dahl J, et al. Predictors of survival from out-of-hospital cardiac arrest: a systematic review and meta-analysis Circ Cardiovasc Qual Outcomes 2010;3:63-81.

3. Cardiopulmonary resuscitation: statement by the Ad Hoc Committee on Cardiopulmonary Resuscitation of the Division of Medical Sciences, National Academy of Sciences-National Research Council. JAMA 1966;198:372-9.

4. Emergency Cardiac Care Committee and Subcommittees AHA. Guidelines for cardiopulmonary resuscitation and emergency cardiac care. JAMA 1992;268:2171-302.

5. Weisfeldt ML, Becker LB. Resuscitation after cardiac arrest: a 3-phase time-sensitive model. JAMA 2002;288:3035-8.

6. Resuscitation ILCo. 2005 international consensus on cardiopulmonary resuscitation and emergency cardiovascular care science with treatment recommendations. Resuscitation 2005;293:181-341.

7. Aufderheide TP, Sigurdsson G, Pirrallo RG, et al. Hyperventilationinduced hypotension during cardiopulmonary resuscitation. Circulation 2004;109:1960-5.

8. Aufderheide TP, Lurie KG. Death by hyperventilation: a common and life-threatening problem during cardiopulmonary resuscitation. Crit Care Med 2004;32:S345-51.

9. Ewy GA. Cardiocerebral resuscitation: the new cardiopulmonary resuscitation. Circulation 2005;111:2134-42.

10. Kern KB, Valenzuela TD, Clark LL, et al. An alternative approach to advancing resuscitation science. Resuscitation 2005;64:261-8.

11. Rea TD, Helbock M, Perry S, et al. Increasing use of cardiopulmonary resuscitation during out-of-hospital ventricular fibrillation arrest: Survival implications of guideline changes. Circulation 2006;114:2760-5

12. Ewy GA, Kern KB. Recent advances in cardiopulmonary resuscitation: cardiocerebral resuscitation. J Am Coll Cardiol 2009;53:149-57.

13. Stroup DF, Berlin JA, Morton SC, et al. Meta-analysis of observational studies in epidemiology: a proposal for reporting. Meta-analysis Of Observational Studies in Epidemiology (MOOSE) group. JAMA 2000;283:2008-12.

14. Boehm K, Borelli F, Ernst E, et al. Green tea (Camellia sinensis) for the prevention of cancer. Cochrane Database Syst Rev 2009;(3): CD005004. doi:10.1002/14651858.CD005004.pub2.

15. Wells GA, Shea B, O'Connell D, et al. The Newcastle-Ottawa Scale (NOS) for assessing the quality of nonrandomised studies in metaanalyses. University of Ottawa, http://www.Iri.ca/programs/ceu/ oxford.htm. 2012; (accessed April 2012).

16. DerSimonian R, Laird N. Meta-analysis in clinical trials. Control Clin Trials 1986;7:177-88.

17. Review Manager (RevMan) [Computer program]. Version 5.1 Copenhagen: The Nordic Cochrane Centre TCC, 2011.

18. Bobrow BJ, Clark LL, Ewy GA, et al. Minimally interrupted cardiac resuscitation by emergency medical services for out-of-hospital cardiac arrest. JAMA 2008;299:1158-65.

19. Garza AG, Gratton MC, Salomone JA, et al. Improved patient survival using a modified resuscitation protocol for out-of-hospital cardiac arrest. Circulation 2009;119:2597-605.

20. Kellum MJ, Kennedy KW, Barney R, et al. Cardiocerebral resuscitation improves neurologically intact survival of patients with out-of-hospital cardiac arrest. Ann Emerg Med 2008;52:244-52.

21. Aufderheide T, Yannopoulos D, Lick CJ, et al. Implementing the 2005 American Heart Association Guidelines improves outcomes after out-of-hospital cardiac arrest. Heart Rhythm 2010;7:1357-62.

22. Bigham B, Koprowicz K, Rea T, et al. Cardiac arrest survival did not increase in the Resuscitation Outcomes consortium after implementation of the 2005 AHA CPR and ECC guidelines. Resuscitation 2011;81:979-83.

23. Hinchey P, Myers JB, Lewis R, et al. Improved out-of-hospital cardiac arrest survival after the sequential implementation of $2005 \mathrm{AHA}$ guidelines for compressions, ventilations, and induced hypothermia: the Wake County experience. Ann Emerg Med 2010;56:348-57.

24. Hung SW, Chen $\mathrm{C}$, Shih $\mathrm{HC}$, et al. Are new resuscitation guidelines better? Experience of an Asian metropolitan hospital. Ann Acad Med Singapore 2010;39:569-75.

25. Lick C, Aufderheide TP, Niskanen RA, et al. Take Heart America: a comprehensive, community-wide, systems-based approach to the treatment of cardiac arrest. Crit Care Med 2011;39:26-33.

26. Olasveengen TM, Vik E, Kuzovlev A, et al. Effect of implementation of new resuscitation guidelines on quality of cardiopulmonary resuscitation and survival. Resuscitation 2009;80:407-11.

27. Robinson S, Swain AH, Hoyle SR, et al. Survival from out-of-hospital cardiac arrest in New Zealand following the 2005 resuscitation guideline changes. Resuscitation 2010;81:1648-51.

28. Sayre MR, Cantrell SA, White LJ, et al. Impact of the 2005 American Heart Association cardiopulmonary resuscitation and emergency 
cardiovascular care guidelines on out-of-hospital cardiac arrest survival. Prehosp Emerg Care 2009;13:469-77.

29. Steinmetz J, Barnung S, Nielsen SL, et al. Improved survival after an out-of-hospital cardiac arrest using new guidelines. Acta Anaesthesiol Scand 2008;52:908-13.

30. Ioannidis JP. Interpretation of tests of heterogeneity and bias in meta-analysis. J Eval Clin Pract 2008;14:951.

31. Sanders AB, Kern KB, Atlas M, et al. Importance of the duration of inadequate coronary perfusion pressure on resuscitation from cardiac arrest. J Am Coll Cardiol 1985;6:113-18.

32. Kern KB, Ewy GA, Voorhees WD, et al. Myocardial perfusion pressure: a predictor of 24-hour survival during prolonged cardiac arrest in dogs. Resuscitation 1988;16:241-50.

33. Cobb LA, Fahrenbruch CE, Walsh TR, et al. Influence of cardiopulmonary resuscitation prior to defibrillation in patients with out-of-hospital ventricular fibrillation. JAMA 1999;281: $1182-8$.

34. Berg RA, Hilwig RW, Ewy GA, et al. Precountershock cardiopulmonary resuscitation improves initial response to defibrillation from prolonged ventricular fibrillation: a randomized, controlled swine study. Crit Care Med 2004;32:1352-7.

35. Niemann JT, Cairns CB, Sharma J, et al. Treatment of prolonged ventricular fibrillation. Immediate countershock versus high-dose epinephrine and CPR preceding countershock. Circulation 1992;85:281-7.

36. Chamberlain D, Freneaux M, Steen $\mathrm{S}$, et al. Why do chest compressions aid defibrillation? Resuscitation 2008;77:10-15.
37. Meier $\mathrm{P}$, Baker $\mathrm{P}$, Jost $\mathrm{D}$, et al. Chest compressions before defibrillation for out-of-hospital cardiac arrests: a meta-analysis of randomized controlled clinical trials. BMC Med 2010;8:52-63.

38. Wang HE, Simeone SJ, Weaver MD, et al. Interruptions in cardiopulmonary resuscitation from paramedic endotracheal intubation. Ann Emerg Med 2009:54:645-52.

39. Bobrow BJ, Ewy GA, Clark L, et al. Passive oxygen insufflation is superior to bag-valve-mask ventilation for witnessed ventricular fibrillation out-of-hospital cardiac arrest. Ann Emerg Med 2009;54:656-62.

40. Fletcher D, Chamberlain D, Handley A, et al. Utstein-style audit of Protocol C: a non-standard resuscitation protocol for healthcare professionals. Resuscitation 2011;82:1265-72.

41. Deakin C, Nolan J, Soar J, et al. European Resuscitation Council Guidelines for Resuscitation 2010 Section 4. Adult advanced life support. Resuscitation 2010;81:1305-52.

42. Berg RA, Hemphill R, Abella BS, et al. Part 5: adult basic life support: 2010 American Heart Association Guidelines for Cardiopulmonary Resuscitation and Emergency Cardiovascular Care. Circulation 2010;122:S685-705.

43. Bernard SA, Gray T, Buist MD, et al. Treatment of comatose survivors of out-of-hospital cardiac arrest with induced hypothermia. New Engl J Med 2002;346:557-63.

44. Cheung KW, Green RS, Magee KD. Systematic review of randomized controlled trials of therapeutic hypothermia as a neuroprotectant in post cardiac arrest patients. Can J Emerg Med 2006;8:329-37. 


\section{Correction}

Salmen M, Ewy GA, Sasson C. Use of cardiocerebral resuscitation or AHA/ERC 2005 Guidelines is associated with improved survival from out-of-hospital cardiac arrest: a systematic review and meta-analysis. BMJ Open 2012;2:e001273.

Figure 1 in this article was mislabelled and should have been: "Figure 1 AHA versus ERC versus cardiocerebral resuscitation characteristics." Figure 2 should have been: "Figure 2 Flow chart depicting the outline of study selection process." Figure 3 should have been: "Figure 3 Forest plot of pooled ORs: cardiocerebral resuscitation versus AHA 2000 studies.

In addition, the second sentence of the first full paragraph of page 5 contains an error. It is stated "Pooled OR of survival to hospital discharge for this subgroup was 2.98 (5\% CI 1.92 to 4.62) (figure 3))". In fact, this needs to be "(figure 4)", and not the figure 4 that is currently included in the manuscript. The correct version of figure 4 is below. The correct label should be "Figure 4 Forest plot of ORs for witnessed ventricular fibrillation/ventricular tachycardia survival: cardiocerebral resuscitation versus AHA/ERC 2000 studies." The second paragraph on the fifth page, left column, should then read: "Eight of the nine studies using AHA/ERC 2005 Guidelines demonstrated improved survival to hospital discharge for use of AHA/ERC 2005 compared to AHA/ERC 2000 Guidelines, a result that was statistically significant in five out of nine studies (figure 5)."

We apologise for these errors.

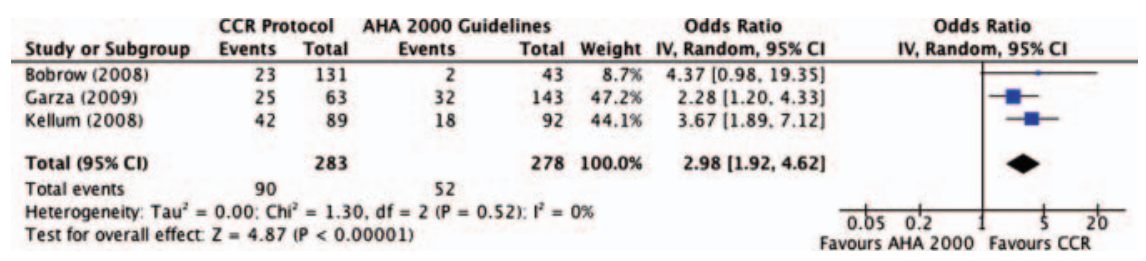

BMJ Open 2012;2:e001273corr1. doi:10.1136/bmjopen-2012-001273corr1 